\title{
Correction to: SEOM clinical guidelines for anaplastic gliomas (2017)
}

\author{
C. Balañá ${ }^{10} \cdot$ M. Alonso ${ }^{2} \cdot$ A. Hernandez-Lain ${ }^{3} \cdot$ P. Perez-Segura ${ }^{4}$ E. Pineda ${ }^{5} \cdot$ A. Ramos ${ }^{3} \cdot$ A. R. Sanchez ${ }^{6}$. \\ P. Teixidor ${ }^{7} \cdot$ E. Verger ${ }^{5} \cdot$ M. Benavides ${ }^{8}$
}

Published online: 1 June 2018

๑) Federación de Sociedades Españolas de Oncología (FESEO) 2018

\section{Correction to: Clin TransI Oncol (2018) 20:16-21 https://doi.org/10.1007/s12094-017-1762-7}

Unfortunately the name of one of the authors was spelled incorrectly in the published original article. The correct name is A. Hernandez-Lain instead of A. Hernandez.

The original article can be found online at https://doi.org/10.1007/ s12094-017-1762-7.

C. Balañá

cbalana@iconcologia.net

M. Alonso

miriamag3@hotmail.com

A. Hernandez-Lain

aurelioneu@yahoo.es

P. Perez-Segura

perezsegura09@gmail.com

E. Pineda

epineda@clinic.cat

A. Ramos

ramosana3@yahoo.es

\section{A. R. Sanchez}

alossantos@gmail.com

P. Teixidor

pilarteixidor.germanstrias@gencat.cat

E. Verger

EVERGER@clinic.ub.es
M. Benavides

manuel.benavides.sspa@juntadeandalucia.es

1 Institut Català Oncologia Badalona, Ct. Canyet, s/n, 08916 Barcelona, Spain

2 Complejo Hospitalario Virgen del Rocío, Seville, Spain

3 Hospital 12 de Octubre, Madrid, Spain

4 Hospital Universitario Clínico San Carlos, Madrid, Spain

5 Hospital Clínic i Provincial, Barcelona, Spain

6 Complejo Asistencial Universitario de León, León, Spain

7 Hospital Universitari Germans Trias i Pujol Badalona, Barcelona, Spain

8 Hospital Universitario Regional y Virgen de la Victoria, Málaga, Spain 